\title{
VISCOUS HYPERELASTIC MATERIALS MODELING
}

\author{
SEMENOV A. $\mathrm{V}^{1}$, NASONOVSKY N. $\mathrm{P}^{2}$, SMIRNOV A. $\mathrm{V}^{3}$, STEPANOV M. $\mathrm{D}^{4}$, KHARALDIN N. $\mathrm{A}^{5}$, \\ STEPANOV A.V $\mathbf{V}^{6} \&$ BOROVKOV A. $\mathbf{I}^{7}$ \\ 1,2,3,4,5,6 Center of National Technological Initiative, Engineering Center “Center of Computer-Aided Engineering” Peter the \\ Great St.Petersburg Polytechnic University St. Petersburg, Russia \\ ${ }^{7}$ Professor, vice-rector for innovative projects of Peter the Great St. Petersburg, Center of National Technological Initiative, \\ Engineering Center “Center of Computer-Aided Engineering” Peter the Great St.Petersburg Polytechnic University \\ St. Petersburg, Russia
}

\begin{abstract}
This article is about the analysis and choice of the computer modeling optimal method of viscous hyperelastic materials in the LS-Dyna for solving tasks that meet contemporary requirements. The main models of hyperelastic materials implemented in the LS-Dyna numerical analysis software package were considered, which made it possible to make the stress-strain analysis based on deformation rate. The process of experimental data adaptation for using in MAT_077_OGDEN_RUBBER and MAT_181_SIMPLIFIED_RUBBER materials models is described.

KEYWORDS: Computer Modeling, Finite-Element Method, Material Model, Elastomer, Hyperelasticity \& Viscosity
\end{abstract}

Received: Jun 08, 2020; Accepted: Jun 28, 2020; Published: Sep 25, 2020; Paper Id.: IJMPERDJUN20201425

\section{INTRODUCTION}

In engineering practice there are often hyperelastic materials structural analysis problems. Mechanical-and-physical properties of such materials can vary significantly depending on the speed of external mechanical impact. This must be considered in situations with velocity variation wide range. Choosing and adjusting a material model can have a significant impact on the result.

The implementation of the virtual tests described in the work was carried out on the basis of the platform CML-BenchTM (digital platform for the development of digital twins and an activity management system in the field of digital design, mathematical modeling and computer engineering, development of the engineering center CEC SPbPU) using commercial licenses LS-DYNA and computational resources of CNTI.

\section{REFERENCE DATA}

\section{A. Fundamental Definition}

The mechanical properties of an isotropic hyperelastic material are described by strain energy function, as a function of three invariants of the Cauchy-Green strain tensor [1, 2] 
$W=f\left(I_{1}, I_{2}, I_{3}\right)$

$\mathrm{W}$ is the strain energy density, I_1, I_2, I_3 are three invariants of the Cauchy-Green strain tensor described by three main elongations.

$$
\begin{aligned}
& I_{1}=\lambda_{1}^{2}+\lambda_{2}^{2}+\lambda_{3}^{2}, \\
& I_{2}=\lambda_{1}^{2} \lambda_{2}^{2}+\lambda_{2}^{2} \lambda_{3}^{2}+\lambda_{1}^{2} \lambda_{3}^{2} \\
& I_{3}=\lambda_{1}^{2} \lambda_{2}^{2} \lambda_{3}^{2} \\
& I \_3=1 \text { for incompressible materials. }
\end{aligned}
$$

Elongation is defined below:

$\lambda=\frac{L}{L_{0}}=1+\varepsilon$,

where $\mathrm{L}$ is the testing length after strain; $\mathrm{L} 0$ is the testing length before strain; $\varepsilon$ is the elastic strain.

The strain energy is related to the principal stress of the Cauchy tensor as follows:

$$
\square_{i}=\lambda_{i} \frac{\partial W}{\partial \lambda_{i}}
$$

$\mathrm{W}$ is the strain energy.

There is a connection between Cauchy stress (true stress) and Piola-Kirchhoff stress tensor (engineering stress):

$$
\square_{i}^{e n g}=\frac{\sigma_{i}}{\lambda_{i}}
$$

Let us have a look at analytical models of the strain energy function of a hyperelastic material. These models will be used to approximate experimental data and to get environmental parameters.

\section{B. Mooney Rivlin Model}

The Mooney Rivlin model defines the mechanical strain energy as the sum of invariants as following [3-5]]

$$
W=\sum_{i} \quad \sum_{j} \quad C_{i j}\left(I_{1}-3\right)^{i}\left(I_{2}-3\right)^{j}+D(J-1)^{2},
$$

where $\mathrm{J}$ is the relative volume change; $\mathrm{C}_{-} \mathrm{ij}$ and $\mathrm{D}$ are material constants. of sample.

The constants $\mathrm{C}_{-} \mathrm{ij}$ and $\mathrm{D}$ are determined by approximating the experimental data obtained under uniaxial tension

For incompressible material $[6,7]$

$$
\begin{aligned}
& W=\sum_{i} \quad \sum_{j} \quad C_{i j}\left(I_{1}-3\right)^{i}\left(I_{2}-3\right)^{j} \\
& { }_{1} \lambda_{2} \lambda_{3}=1
\end{aligned}
$$

The main values of the Cauchy stress tensor in this case are defined as:

$$
{ }_{i}=\lambda_{i} \frac{\partial W}{\partial \lambda_{i}}-p=\lambda_{i}\left(\frac{\partial W}{\partial I_{1}} \frac{\partial I_{1}}{\partial \lambda_{i}}+\frac{\partial W}{\partial I_{2}} \frac{\partial I_{2}}{\partial \lambda_{i}}\right)-p
$$

The difference of the main Cauchy stress:

$$
\sigma_{1}-\sigma_{3}=\lambda_{1} \frac{\partial W}{\partial \lambda_{1}}-\lambda_{3} \frac{\partial W}{\partial \lambda_{3}}
$$

For uniaxial tension of an incompressible material [8]: 


$$
\begin{aligned}
& \square_{2}=\sigma_{3}=0 \\
& \square_{1}=\lambda \\
& \square_{2}=\lambda_{3}=\frac{1}{\sqrt{\lambda}} \\
& I_{1}=\lambda^{2}+\frac{2}{\lambda} \\
& I_{2}=2 \lambda+\frac{1}{\lambda^{2}}
\end{aligned}
$$

Let's have a look at the two-parameter Mooney-Rivlin model.

The main Cauchy stresses are:

$$
\square_{1}-\sigma_{3}=2 C_{10}\left(\lambda_{1}^{2}-\lambda_{3}^{2}\right)+2 C_{01}\left(\frac{1}{\lambda_{3}^{2}}-\frac{1}{\lambda_{1}^{2}}\right)
$$

As ${ }_{3}=0,{ }_{1}=\lambda, \quad 3=\frac{1}{\sqrt{\lambda}}$, then

$\square_{1}=2 C_{10}\left(\lambda^{2}-\lambda^{-1}\right)+2 C_{01}\left(\lambda-\lambda^{-2}\right)$

Or

$$
\sigma_{1}=\left(2 C_{10}+\frac{2 C_{01}}{\lambda}\right)\left(\lambda^{2}-\lambda^{-1}\right)
$$

Going from true stress to engineering one we get:

$$
\square_{1}^{e n g}=2\left(C_{10}+\frac{C_{01}}{\lambda}\right)\left(\lambda-\lambda^{-2}\right)
$$

Similarly for the Mooney-Rivlin model with five parameters.

\section{Ogden Model}

The Ogden model determines the mechanical strain energy based on principal elongations [9]

$$
W=\sum_{p=1}^{n} \frac{\mu_{p}}{\alpha_{p}}\left(\lambda_{1}^{\alpha_{p}}+\lambda_{2}^{\alpha_{p}}+\lambda_{3}^{\alpha_{p}}-3\right)
$$

Doing the same for the Ogden model as we did for the Mooney-Rivlin model we get the engineering stress for uniaxial tension in the following form:

$$
\square_{\text {eng }}=\sum_{p=1}^{N} \quad \mu_{p}\left(\lambda^{\alpha_{p}-1}-\lambda^{-\frac{\alpha_{p}}{2}-1}\right)
$$

where $\mu \_p$ and $\alpha \_p$ are the material constants.

\section{Viscosity}

The strain rate dependence is determined by entering in addition to the hyperelastic model a viscoelastic component in the following form $[10,11]$ :

$$
\square_{i}(t)=\sigma_{i}^{e n g}+\int_{0}^{t} \quad G(t-\tau) \frac{\partial E_{i}}{\partial \tau} d \tau ;
$$

$\mathrm{G}(\mathrm{t}-\tau)$ is the relaxation process. 


$$
E_{i}=\frac{1}{2}\left(\lambda_{i}^{2}-1\right) ; G(t)=\sum_{n=1}^{N} \quad G_{n} e^{-\beta_{n} t}
$$

The relaxation function is shown in Prony series [10].

$$
\int_{0}^{t} \quad G(t-\tau) \frac{\partial E_{i}}{\partial \tau} d \tau=\sum_{n=1}^{N} \quad G_{n} \int_{0}^{t} \quad e^{-\beta_{n}(t-\tau)} \lambda_{i} \frac{\partial \lambda_{i}}{\partial \tau} d \tau
$$

\section{PHYSICOMECHANICAL PARAMETERS OF THE MATERIAL}

SKU-PFL-100 is a two-component polyurethane with SKU-PFL-100 urethane prepolymer and Diameter X hardener.

As the basic data for SKU-PFL-100 some physical and mechanical parameters of material are listed in Table 1, the static loading curve in the strain range $[-0.75 ; 3.5]$, as well as curves for various strain rates in the compression section [-0.3; 0]. [12]

Table 1: Physicomechanical Characteristics

\begin{tabular}{|l|c|}
\hline \multicolumn{1}{|c|}{ Characteristics } & SKU-PFL-100 \\
\hline Density, $\frac{g}{s m^{3}}$ & 1.12 \\
\hline Tensile strength, MPA & $30-50$ \\
\hline Elastic constant, MPA & $20-23$ \\
\hline Extension elongation, \% & $380-400$ \\
\hline Tensile failure, \% & $4-10$ \\
\hline
\end{tabular}

A solid cylinder $30 \times 26.5 \mathrm{~mm}$ was used as a sample to get a compressibility data. A blade type 1 was used according to GOST 270-75 was used for stretching, $7 \mathrm{~mm}$ wide, $2 \mathrm{~mm}$ thick, measuring base $25 \mathrm{~mm}$.

\section{VISCOUS HYPERELASTIC MATERIALS IN LS-DYNA MATHEMATICAL MODEL}

LS-DYNA has several mathematical models in its functionality that describe the behavior of elastomers [13].

In this article we will describe MAT_077_OGDEN_RUBBER and MAT_181_SIMPLIFIED_RUBBER.

\section{A. MAT_077}

To describe the behavior of the material using the MAT_077 model, it is necessary to set the material density, Poisson's ratio, as well as the parameters of the Mooney-Rivlin model (MAT_077_H) or the parameters of the Ogden model (MAT_077_O). For an incompressible material the Poisson's ratio is 0.5 , but this value will cause problems in the numerical scheme, therefore, a close value of 0.49 is set. The material behavior models coefficients can be set manually, or a curve can be loaded for further approximation using the corresponding model.

The MAT_077_O model was chosen, since the MAT_077_H model is unstable when strained above 1.5, which is connected with the Drucker stability criterion [14].

The result of approximating the initial data by the Ogden model is presented in Figure 1. The coefficients are below:

$$
\begin{aligned}
& { }_{1}=0.00627 ; \mu_{2}=35.7 ; \mu_{3}=89.6 ; \\
& { }_{1}=6.91 ; \alpha_{2}=0.933 ; \alpha_{3}=-0.193
\end{aligned}
$$




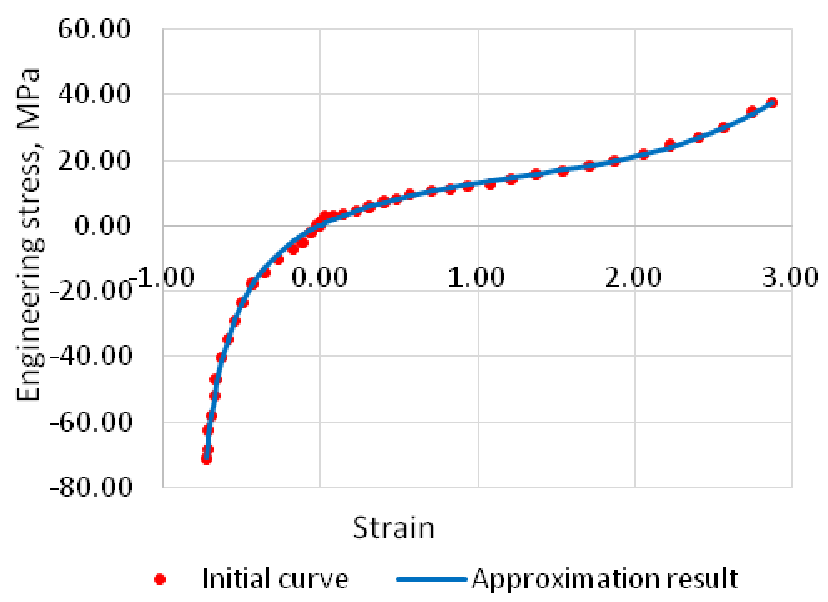

Figure 1: The Result of Initial Data Approximation According to the Ogden Model.

To enter the strain rate dependence it is necessary to set the Prony series coefficients. The input of the coefficients of the first 12 numbers of the Prony series is supported. 3-5 is recommended.

In this article the coefficients of the first two summands are found:

$$
\begin{aligned}
& G_{1}=33.6 ; G_{2}=1.96 ; \\
& ]_{1}=364 ; \beta_{2}=0.1
\end{aligned}
$$

\section{B. MAT_181}

To describe the behavior of the material using the * MAT_181 model it is necessary to determine the following variables $[15,16]$ :

- The density of the material RO;

- Volume modulus of elasticity KM;

- Damping coefficient MU;

- Strain curve LC / TBID;

- Material strain characteristics - GAMA1, GAMA2, K.

The loading process is determined either by a single curve or by a family of curves for the strain rate discrete values. The strain curve can be defined as the dependence of the force on absolute elongation. If the SGL, SW and ST parameters are equal to unity, then the curve is defined as the dependence of engineering stress on strain. Curves should cover the entire expected strain area, including both compressive (negative values) and tensile (positive values) regimes.

When determining a family of curves for different strain rates, we should take into account the following parameters:

TENSION The parameter that controls the strain rate effect processing.

EQ.-1.0: the speed effect is considering in the process of compressive and strain loading, but not in the process of unloading, 
EQ.0.0: the speed effect is considering only for compressive load,

EQ.1.0: The speed effect is considering for strain and compressibility.

RTYPE Preset strain rate type

EQ.0.0: true strain rate

$\dot{\square}_{\text {true }}=\frac{v}{L^{\prime}}$

where $\mathrm{L}$ is the size of the sample at the current time; $\mathrm{v}$ is the speed of external influence.

EQ.1.0: engineering strain rate

$\dot{\square}_{\text {eng }}=\frac{v}{L_{0}}$,

where $L_{-} 0$ is the size of the sample in the initial configuration; $v$ is the speed of external influence.

As a strain criterion in * MAT_181 (SIMLIFIED_RUBBER_WITH_FAILURE), the model proposed by Feng and Hallquist [17] is used.

\section{NUMERICAL SOLUTION WITHOUT VISCOSITY}

Let us check the behavior of the models MAT_077 and MAT_181 without the strain rate. Table 2 shows the data to complete the used material cards. The calculations were performed using the supercomputer of the center "Polytechnic" SPbPU.

Table 2: Material Charts

\begin{tabular}{|l|c|c|}
\hline Characteristics & MAT_181 & MAT_77_O \\
\hline RO, т/MM ${ }^{3}$ & 1.12 & 1.12 \\
\hline KM, mPA & 1000 & - \\
\hline PR & - & 0.49 \\
\hline MU & 0.1 & - \\
\hline G & 750 & 750 \\
\hline SIGF & 0.75 & 0.75 \\
\hline LCID & 15 & - \\
\hline K & 0 & - \\
\hline GAMA1 & 0.02 & - \\
\hline GAMA2 & & SKU-PFL-100 curve \\
\hline
\end{tabular}

\section{A. Tension}

The blade model consists of 4500 hexa elements formulation constant stress solid element (figure 2). Hourglass control $\mathrm{IHQ}=5 \mathrm{QH}=0.05$

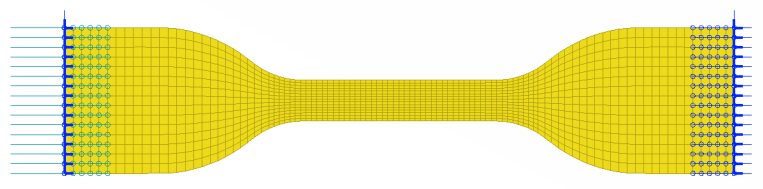

Figure 2: Blade Model. 
The right edge is fixed in all directions, a constant speed is applied to the left edge along the longitudinal axis and movement in other directions is fixed.

The G and SIGF parameters were determined by evaluating their influence on the behavior of the material and adjusting the values accordingly. Table 3 describes the influence of these parameters on the behavior of the material.

Table 3: G and SIGF Influence on MAT_181 Erosion.

\begin{tabular}{|c|c|}
\hline Parameters & Result \\
\hline $\begin{array}{l}\mathrm{G}=0 \\
\mathrm{SIGF}=0\end{array}$ & With large deformations a sharp increase in width and a decrease in thickness occurs. \\
\hline $\begin{array}{l}\mathrm{G}=3.5 \mathrm{e} 4 \\
\mathrm{SIGF}=70\end{array}$ & Incorrect behavior. Necking happens after strain which is common for metals \\
\hline $\begin{array}{l}\mathrm{G}=3500 \\
\mathrm{SIGF}=7\end{array}$ & Vibrations end up quickly after discontinuity \\
\hline $\begin{array}{l}G=1000 \\
S I G F=4\end{array}$ & Vibrations end up quickly after discontinuity \\
\hline $\begin{array}{l}\mathrm{G}=750 \\
\mathrm{SIGF}=0.75\end{array}$ & Vibrations end up mildly \\
\hline
\end{tabular}

Let us compare the loading curves for values $\mathrm{G}=0$, $\mathrm{SIGF}=0 ; \mathrm{G}=1000, \mathrm{SIGF}=4 ; \mathrm{G}=500$, $\mathrm{SIGF}=0.5$ (figure 3-4)

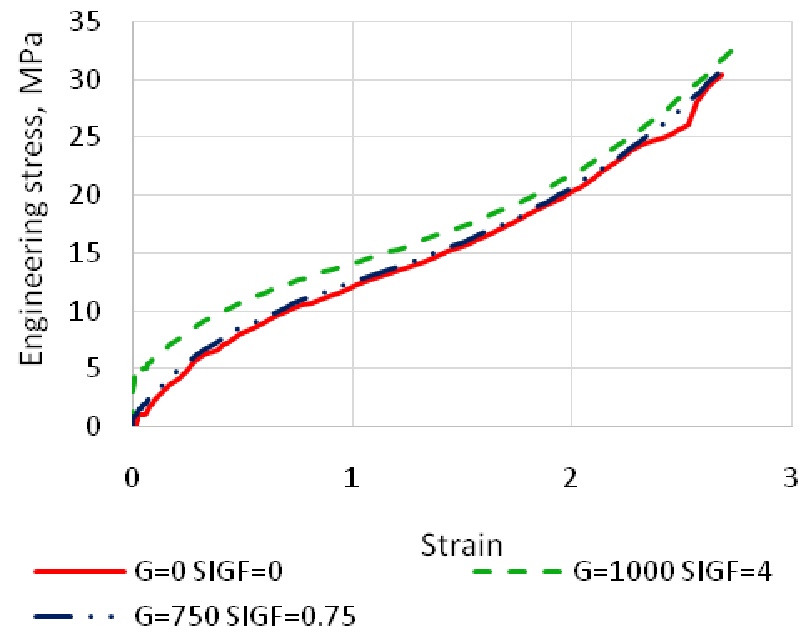

Figure 3: G and SIGF Parameters Influence on MAT_181. 


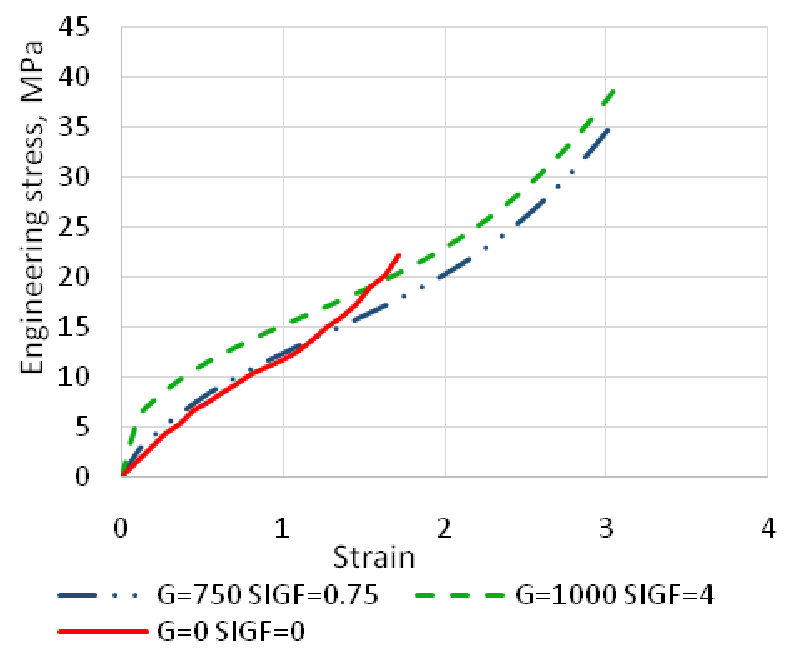

Figure 4: G and SIGF Parameters Influence on MAT_077_O.

As we can see on the graphs, with SIGF parameter increase a stress jump appears at the beginning of the curve. At zero values of the parameters the material is unstable. The minimum value of the SIGF parameter is 0.75 where there is no sharp increase in the cross section of the blade.

The value of $\mathrm{G}$ is selected based on the recommendation $\mathrm{G}=1000 \cdot$ SIGF.

In general, the SIGF and G values must be determined individually for each material, as well as adjusted depending on the strain parameters GAMA1, GAMA2, and K in the MAT_181 model.

The result of a numerical experiment and the removal of the stress-strain curve according to GOST 270-75 shows the discrepancy between the obtained data and the original data at strain values above 1.5.

Moreover, the deviation for MAT_077_O is greater than for MAT_181 (Figure 5).

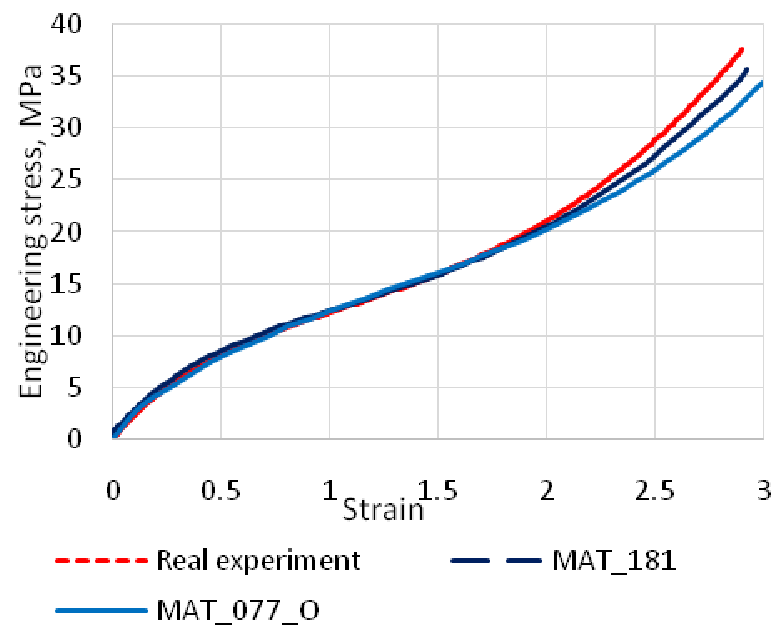

Figure 5: Comparison with a Real Experiment.

The results show that the initial curve is not the truth material loading curve, since it was obtained during the experiment. If a certain elongation is exceeded, strain of areas outside the applied marks begin. Due to the complex blade geometry, these sections have a different sectional area from the one of the narrow part. This gives an estimated faulty proportion of the experiment. 
To get a true material loading curve we have to multiply the original curve by a function

$f(\varepsilon)=a+b e^{\varepsilon}$,

where $\varepsilon$ is the strain; $\mathrm{a}$ and $\mathrm{b}$ are some coefficients.

As an initial approximation, we take the values of the coefficients a and b in such a way so that when we multiply the curve of the numerical experiment it coincides with the curve of the real experiment.

Total coefficients for MAT_181

$a=0,97208396 b=0,00617302$

Total coefficients for MAT_077_O

$a=0,84140297 b=0,04077203$

As a result, the curve of the numerical experiment coincided with the result of a real experiment (Figure 6). Further selection of the coefficients $\mathrm{a}$ and $\mathrm{b}$ is not required.

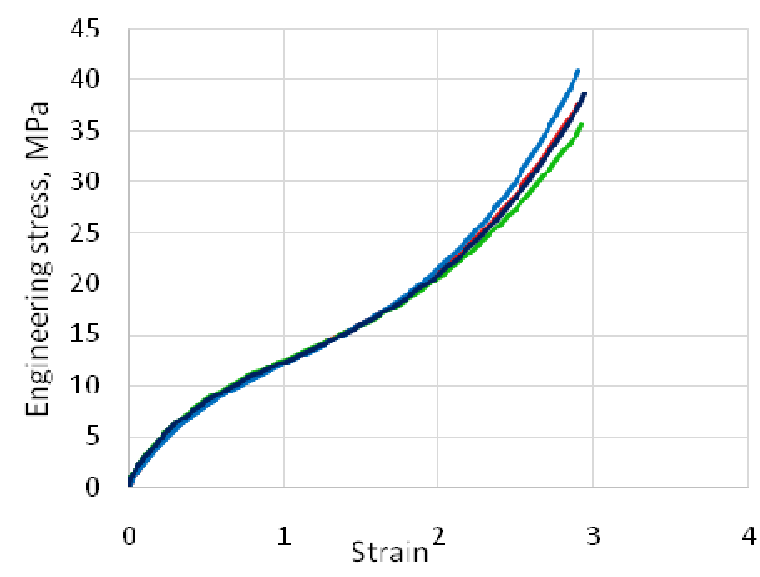

A

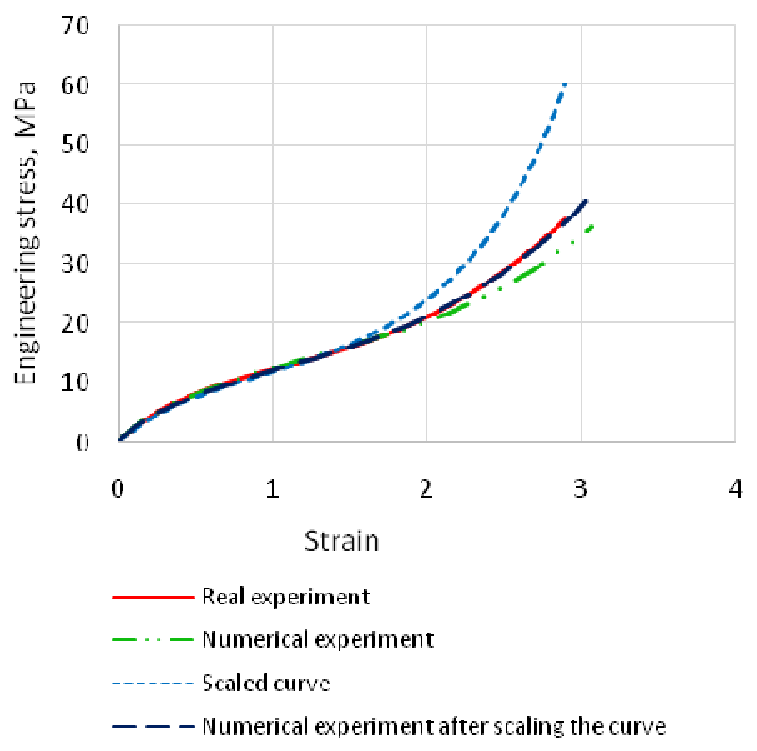

B

Figure 6: Numerical Experiment after Scaling the Curve: A - MAT_181; B - MAT_077. 
Ogden model coefficients for the new curve:

国 ${ }_{1}=0.00435 ; \mu_{2}=47.3 ; \mu_{3}=-46.4$;

$?_{1}=7.69 ; \alpha_{2}=1.17 ; \alpha_{3}=0.885$.

\section{B. Compression}

The cylinder model (figure 7) consists of 16830 hexa elements named as constant stress solid element. Hourglass control $\mathrm{IHQ}=5 \mathrm{QH}=0.05$

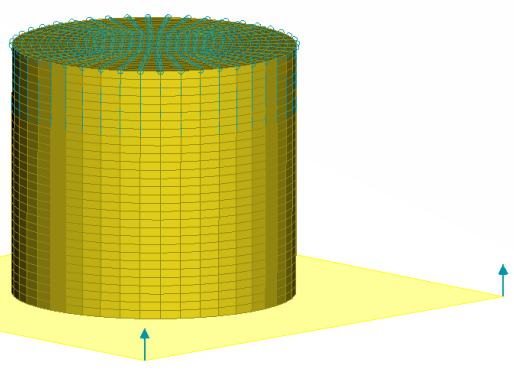

Figure 7: Cylinder Model.

Constant speed is applied to the upper end, the lower end is in contact with * RIGIDWALL_PLANAR

A numerical experiment shows good convergence with the results of a real experiment for MAT_181 (Figure 8). No material parameter adjustments are required.

MAT_077_O shows a slight discrepancy with the original data. This is due to the result of approximation by the Ogden model.

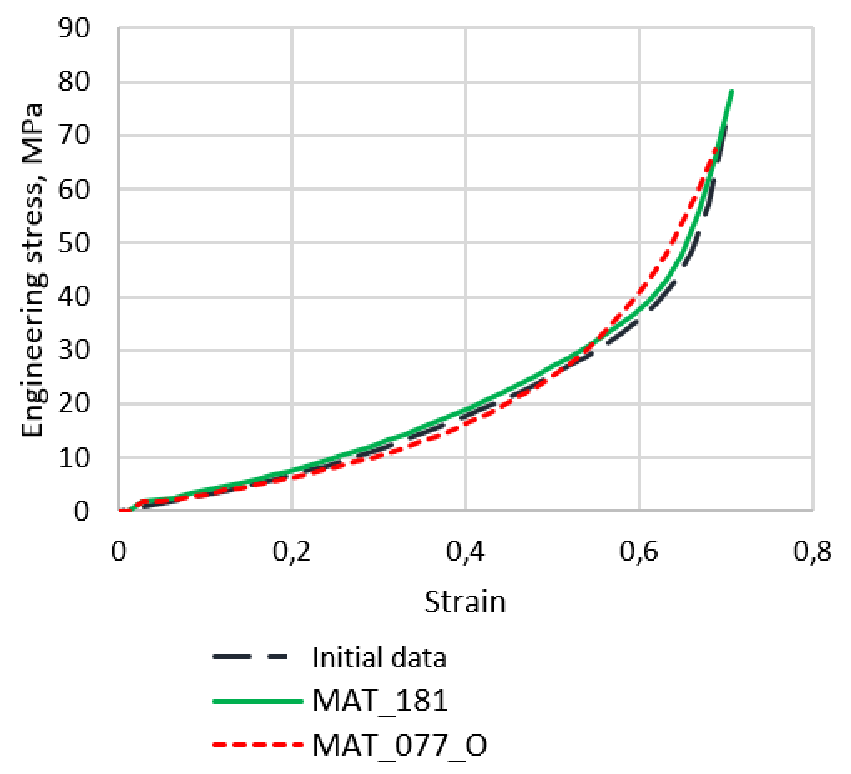

Figure 8: Comparison with a Real Experiment.

\section{The Final Curve of the Material Compared to the Original}

Compared to the original material curve, changes in the tensile area were made in the final curve (figure 9) 


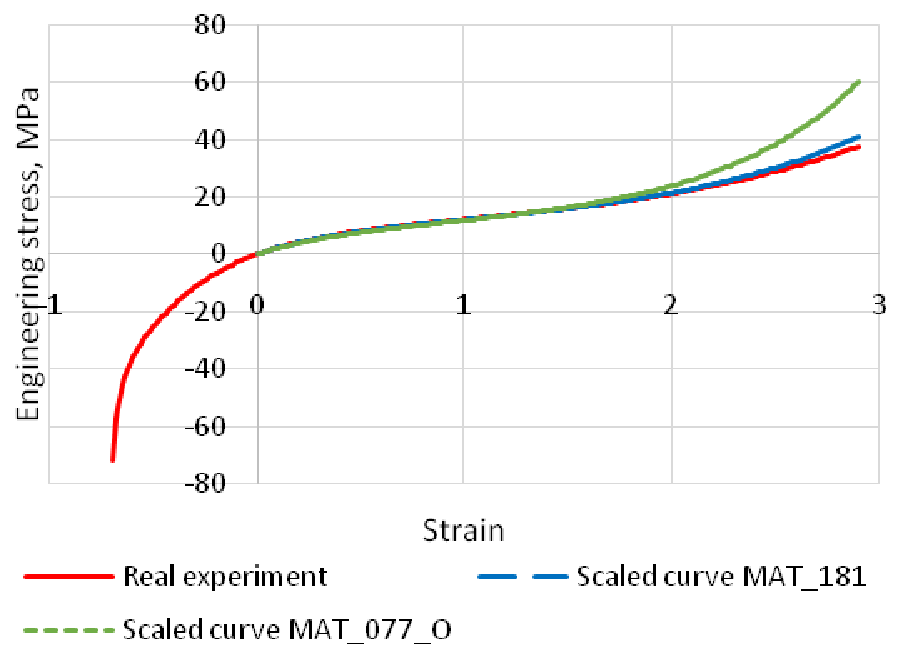

Figure 9: The Original Curve Compared to the Scaled One.

\section{NUMERICAL SOLUTION WITH VISCOSITY}

A family of curves must be defined for different strain rates for MAT_181. Let us use the following scheme:

- To the obtained Mooney-Rivlin material model we add a factor depending on the strain rate. We use the simplest form of a logarithmic dependence on the strain rate

$$
1+a \ln \left(\frac{\dot{\varepsilon}}{b}\right)
$$

- Based on the curves for compression at different strain rates, we get the coefficients of this factor $a=0.1 b=0.04$

The family of curves obtained is shown on figure 10

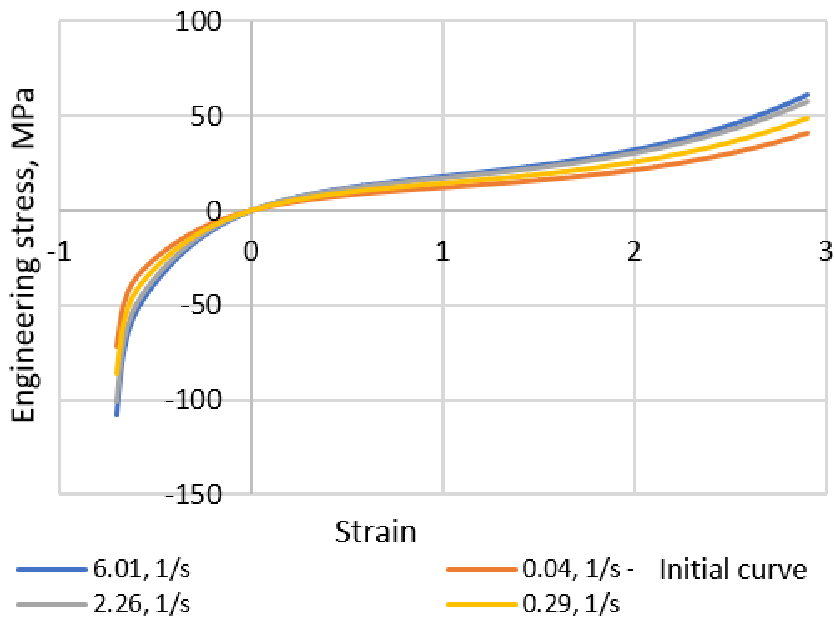

Figure 10: A Family of Curves for MAT_181.

Comparison of curves obtained in known areas is shown in figure 11. 


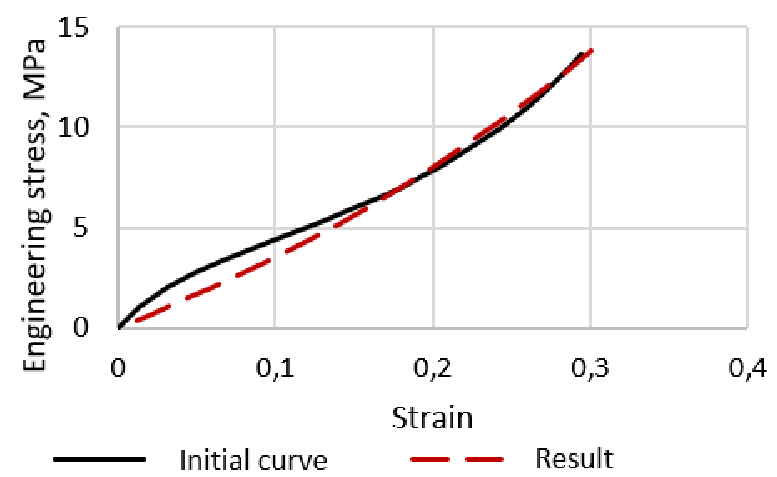

A

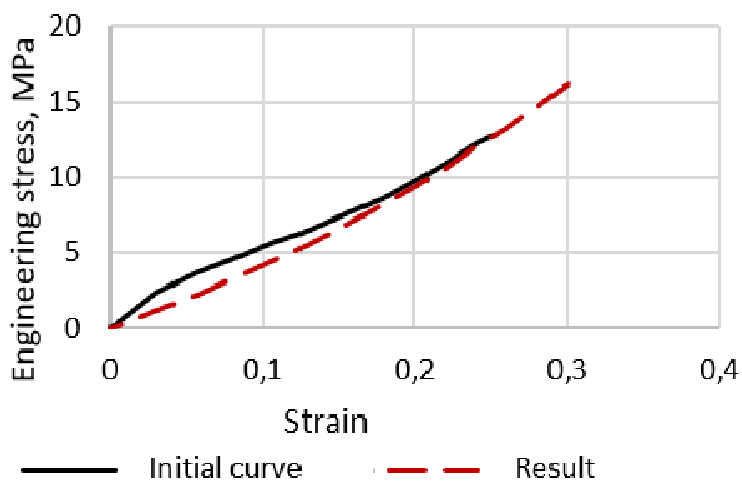

B

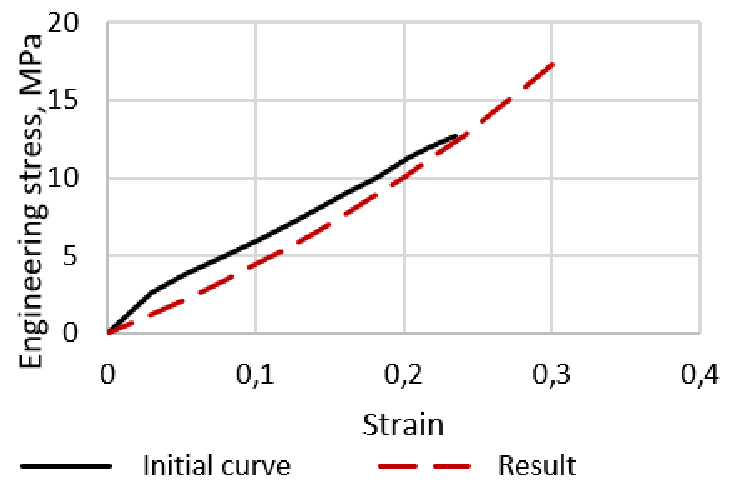

$\mathrm{C}$

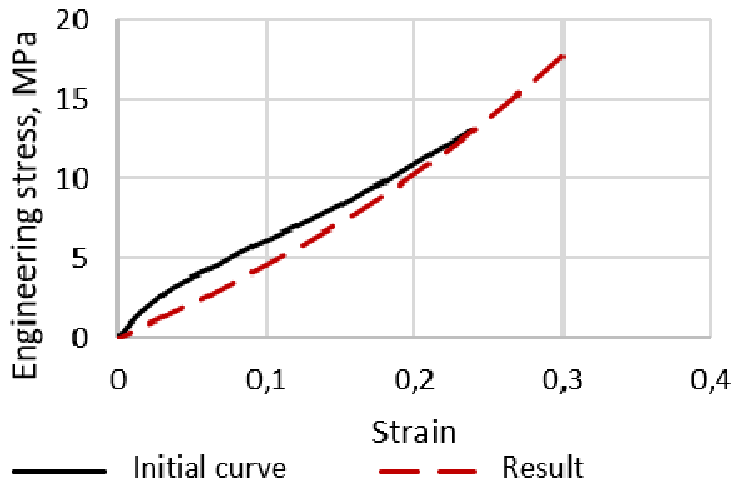

$\mathrm{D}$

Figure 11: Comparison of Curves Obtained on a Known Compression Section.

A - for a Strain Rate of 0.291 / s; B - for a Strain Rate of 2.261 / s;

C - for the Strain Rate of 6.01 1 / s; D - for a Strain Rate of 8.61 1 / s; 


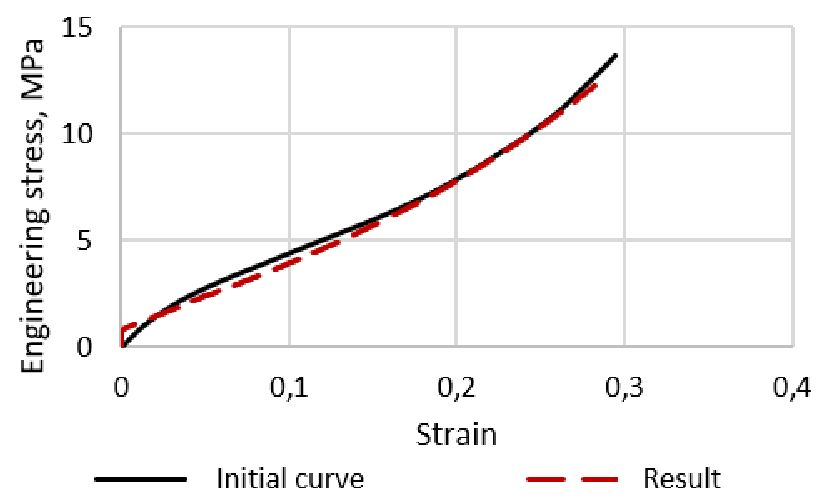

A

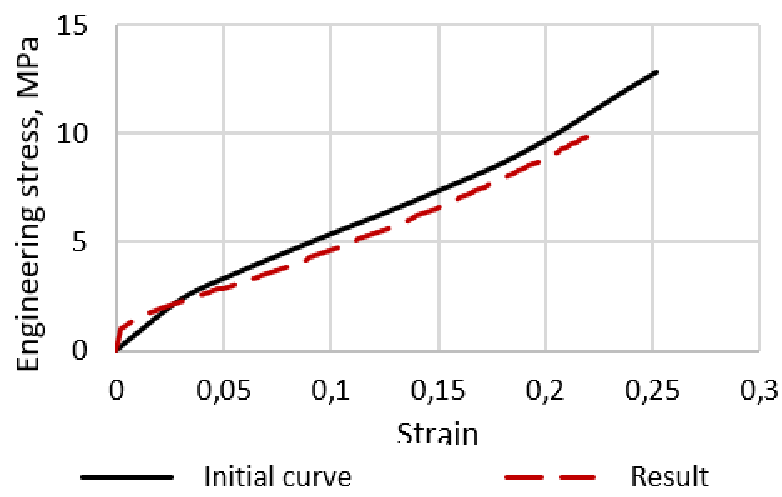

B

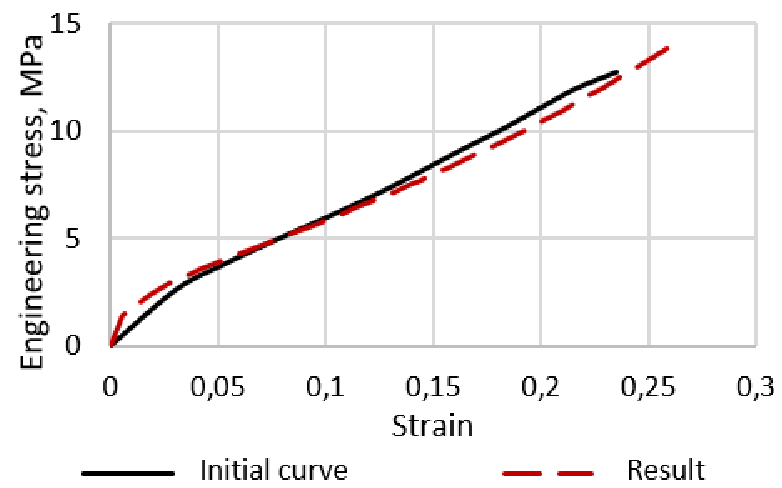

$\mathrm{C}$

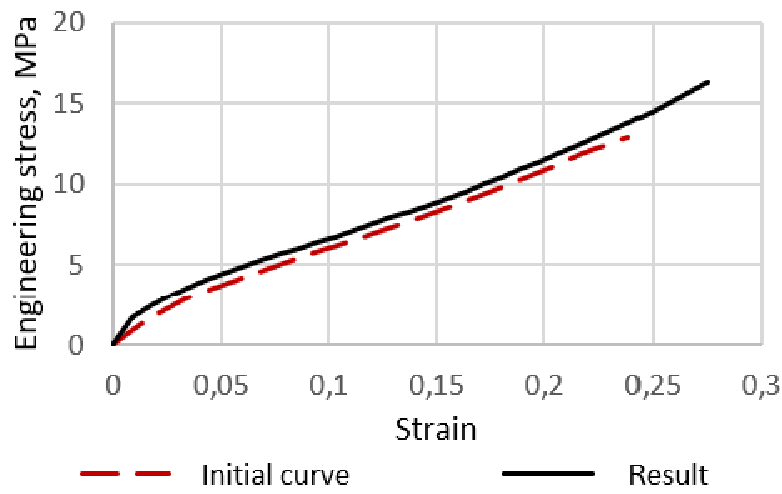

$\mathrm{D}$

Figure 12: Comparison of a Numerical Experiment with a Real one on a Known Compression Area.

$A$ - for a strain rate of $0.291 / \mathrm{s} ; \mathrm{B}$ - for a strain rate of $2.261 / \mathrm{s}$;

$\mathrm{C}$ - for the strain rate of $6.011 / \mathrm{s} ; \mathrm{D}$ - for a strain rate of 8.611 / s; 
For MAT_077_O we add the viscosity parameters obtained earlier. We compare it with the original data for compression at different strain rates (figure 12).

\section{MAT_77 AND MAT_181 COMPARISON}

Let us evaluate the behavior of materials at various strain rates (Figure 13). Let us change the wording of the elements to ELFORM $=-2$. And we also add *CONTROL_ACCURACY OSU=1 INN=4. This was done for more stable material behavior at low strain rates.

MAT_077_O shows stable behavior at both high and low strain rates.

The curves obtained for MAT_181 using the factor (23) differ significantly from the curves obtained by adding viscosity to the MAT_077_O model.

With the current settings, MAT_181 shows unstable behavior after adding curves for different strain rates, especially at low strain rates. No solution to this problem has been found at the moment.

Further studies of the accuracy of the result, as well as a study of the effect of the number of summands of the Prony series on the result obtained are possible only if the results of a tension-compression real experiment carried out at different strain rates are available.

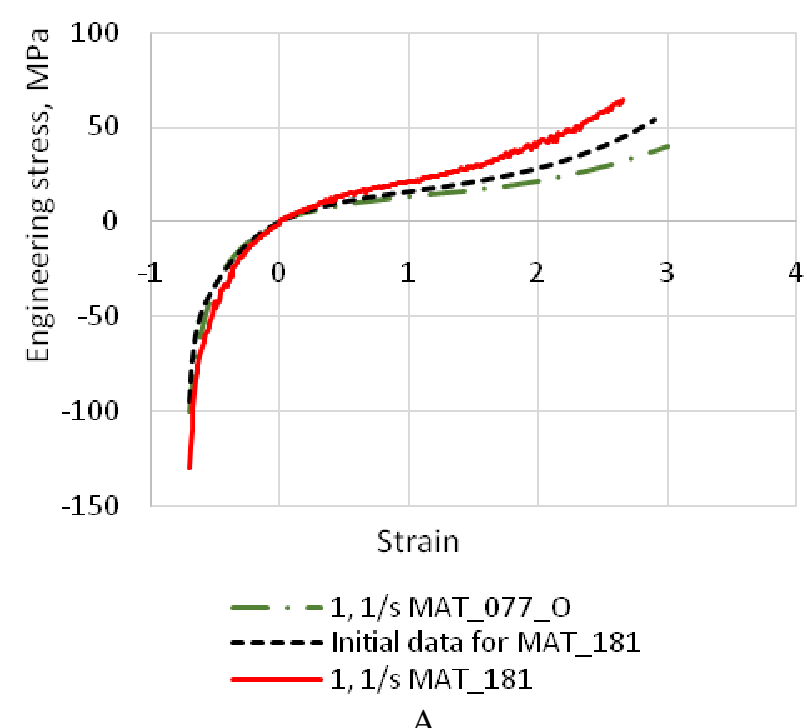

A 


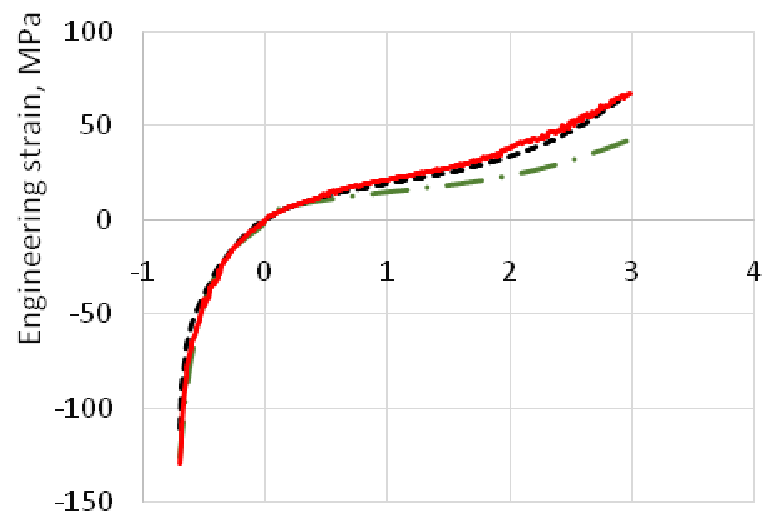

Strain

- - 10,1/s MAT 077

- - - - Initial data for MAT_181

10,1/s MAT_181

$\mathrm{B}$

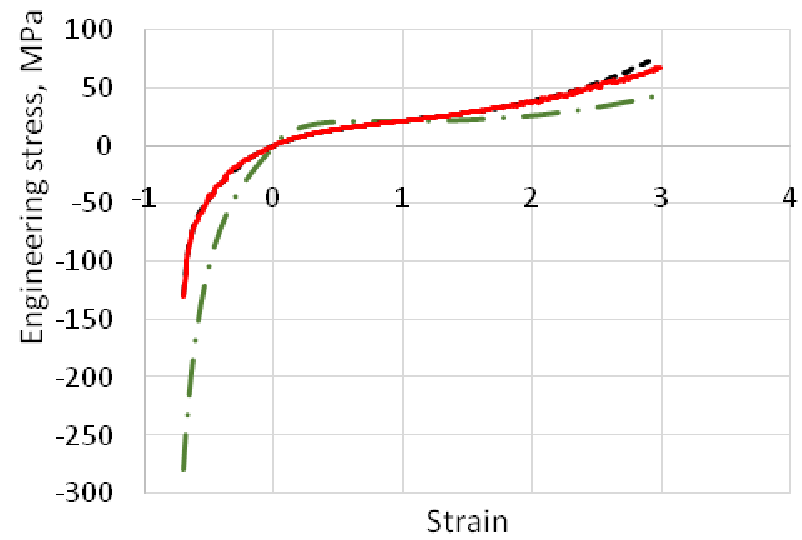

Strain

- $-100,1$ s MAT 077 O

- - - - Initial data for MAT_181

100,1/s MAT_181

$\mathrm{C}$

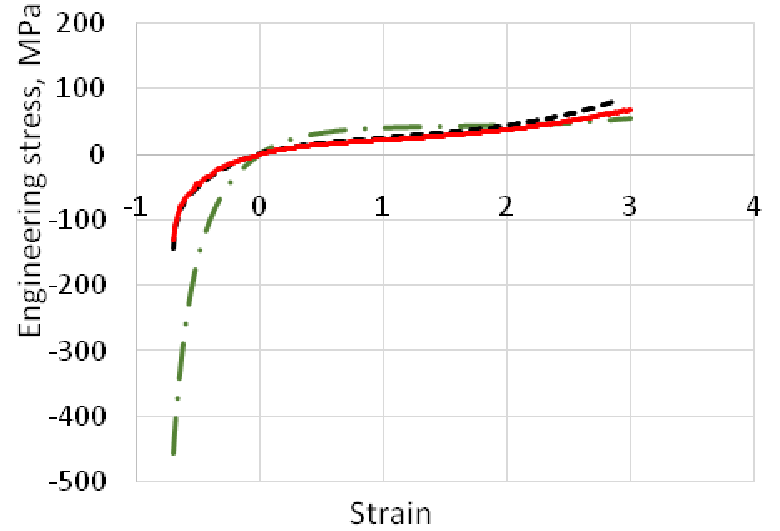

- - $-1000,1 /$ s MAT_077_O

- - - - Initial data for MAT_181

$1000,1 /$ s MAT_181

$\mathrm{D}$

Figure 13: Stress-Strain Curves

A - for the strain rate of 11 / s; B - for strain rate 101 / s;

$\mathrm{C}$ - for the strain rate of 1001 / s; D - for a strain rate of 10001 / s; 


\section{CONCLUSIONS}

In this article we discussed the main models of incompressible hyperelastic materials implemented in the LS-Dyna. Methods of modeling the strain rate influence on the behavior of the material are considered.

Numerical modeling showed similar results when using the MAT_181 and MAT_077 models without considering the dependence on the strain rate. However, when the dependence on the strain rate was introduced for the material model MAT_181 unstable behavior was detected, while MAT_77_O was stable at various strain rates. The viscosity modeling using the Prony series based on the original data demonstrated satisfactory accuracy. However, to further increase the accuracy of the result obtained it is necessary to have more original data, namely, the results of the tensile-compression experiment for various strain rates over the entire work area of the material.

Consequently, it was found that for the numerical modeling of the behavior of hyperelastic material when solving tasks that meet contemporary requirements in the LS-Dyna using the finite element method, it is preferable to use the MAT_077_OGDEN_RUBBER material model because of its stable behavior comparing to the MAT_181 _SIMPLIFIED_RUBBER material model.

\section{REFERENCES}

1. L R G Treloar (1958), The physics of rubber elasticity, Oxford: Clarendon Press, cop., ISBN 978-0-19-857027-1

A. I. Lur'e. Nonlinear Theory of Elasticity. ISBN-13: 978-0444567710

2. Mooney, M., 1940, A theory of large elastic deformation, Journal of Applied Physics, 11(9), pp. 582-592.

3. Rivlin, R. S., 1948, Large elastic deformations of isotropic materials. IV. Further developments of the general theory, Philosophical Transactions of the Royal Society of London. Series A, Mathematical and Physical Sciences, 241(835), pp. 379397.

4. Menderes, H. and Konter, A. W. A., "Advanced FE Analysis of Elastomeric Automobile Components under Realistic Loading Conditions," Proceedings of the First European Conference on Constitutive Models for Rubber, pp. 3-12, 1999

5. Boulanger, P. and Hayes, M. A., 2001, "Finite amplitude waves in Mooney-Rivlin and Hadamard materials", in Topics in Finite Elasticity, ed. M. A Hayes and G. Soccomandi, International Center for Mechanical Sciences.

6. Ogden, R. W., (1972). Large Deformation Isotropic Elasticity - On the Correlation of Theory and Experiment for Incompressible Rubberlike Solids, Proceedings of the Royal Society of London. Series A, Mathematical and Physical Sciences, Vol. 326, No. 1567 (1 February 1972), pp. 565-584.

7. A Comparison Among Neo-Hookean Model, Mooney-Rivlin Model, and Ogden Model for Chloroprene Rubber Beomkeun Kim, Seong Beom Lee, Jayone Lee, Sehyun Cho, Hyungmin Par, Sanghoon Yeom and Sung Han Park

8. C. W. Macosko, 1994, Rheology: principles, measurement and applications, VCH Publishers, ISBN 1-56081-579-5.

9. Christensen, R.M.:A nonlinear theory of viscoelasticity for application to elastomers. J Appl Mech 47(1980) 762-768

10. LS-Dyna. Theory Manual. March 2006. Compiled By. John O. Hallquist

11. A.E. Belkin, I.Z. Dashtiev, B.V. Lonkin Modeling polyurethane viscoelasticity at moderately high strain rates. Mathematical Modeling and Computational Methods, 2014, № 3, pp. 39-54.

12. LS-Dyna Keyword User's Manual Volume II. Material Models. LS-Dyna R11 10/12/18 (r:10572). Livermore Software Technology Corporation (LSTC). 
13. Drucker, D.C. (1959), "A definition of a stable inelastic material", Journal of Applied Mechanics, 26: 101-195

14. “A simplified approach to the simulation of rubber-like materials under dynamic loading” Paul A. Du Bois

15. "Calculation and validation of material tests with specimens made out of filled elastomers" Philipp Thumann, Krzysztof Swidergal, Marcus Wagner

16. W.W. Feng, J.O. Hallquist, A failure criterion for polymers and soft biological materials, 5th European LS-DYNA Users Conference Birmingham Material Technology. (2005). 

